\title{
FORMATION AND EVOLUTION OF GASEOUS BARS
}

\author{
D. FRIEDLI \\ Université Laval and Observatoire du Mt-Mégantic \\ Québec, QC, G1K 7P4, Canada. dfriedli@phy.ulaval.ca
}

\section{Introduction}

Although numerous gaseous bars are observed, they seem to be much less common than stellar bars. This might be due to an observational bias, but gaseous bars could also be difficult to form and/or have short lifetimes. Gas morphology is strongly dependent on the resolution and the considered scale. A true bar-like shape (Maffei 2) should not be confused with either ring-like plus open arms (IC 342), or "twin peaks" (NGC 3351) morphologies. Typical observed gaseous bar characteristics are $2 a_{g} \sim 1 \mathrm{kpc}$, and $M_{g} \sim 0.1-0.3 M_{\mathrm{dyn}}$ inside a few $100 \mathrm{pc}^{1}$. Below, three mechanisms of gaseous bar formation, as well as their evolution, are briefly discussed.

\section{Gaseous Bars in Single-Barred Galaxies}

If the gravitational potential has ILRs, the gas tends to be distributed along nearly circular (partial) rings, like for M100 and NGC 4314, or condensed into twin peaks like for NGC 3351 and NGC 6951 (Kenney 1996). Strong stellar bars without ILRs (e.g. NGC 7479) are able to generate a bar-like morphology for the gas (Laine 1996). In fact, numerical simulations including star formation (Martin \& Friedli 1997) show that young and strong stellar bars host large-scale gaseous bars and " $\mathrm{H} \alpha$ bars". They lead the stellar bar by a few degrees. Moreover, $(b / a)_{g}<(b / a)_{s}, a_{g}<a_{s}, M_{g} \lesssim 10^{9} \mathrm{M}_{\odot}$, and $\Sigma_{g}^{\max } \approx 2.5 \cdot 10^{3} \mathrm{M}_{\odot} \mathrm{pc}^{-2}$. With time, both $a_{g}$ and $M_{g}$ first decrease very quickly, and then reach an asymptotic value; $\tau_{g} \approx 1 \mathrm{Gyr}$. Due to the self-regulation by star formation, $\Sigma_{g}^{\max }$ remains nearly constant.

${ }^{1}$ Definitions: $a_{g}, a_{s}$ and $a_{s e c}=$ semi-major axis of gaseous, primary stellar, and secondary stellar bars; $M_{g}$ and $\Sigma_{g}=$ gas mass and gas surface density; $\tau_{g}=$ gaseous bar lifetime; $M_{\mathrm{dyn}}=$ dynamical mass. 


\section{Gaseous Bars in Double-Barred Galaxies}

Many galaxies host two nested and misaligned (with no systematic angle) stellar bars, often separated by a blue ring. Prototypes are NGC 5850, or NGC 6782 (Wozniak et al. 1995). In these systems, both bars have probably different pattern speeds (Friedli \& Martinet 1993). Numerical simulations (Friedli \& Martinet 1997) indicate a highly time-dependent morphology of the nuclear gaseous ring. Gas is either nearly circular (secondary bar \| primary bar), or strongly concentrated into "twin peaks" (secondary bar $\perp$ primary bar). Inside the secondary bar, a gaseous bar might be present as observed in NGC 2782 (Jogee et al. 1997). In this type of model, $(b / a)_{g}<$ $(b / a)_{\mathrm{sec}}, a_{g}<a_{\mathrm{sec}}, M_{g} \lesssim 4 \cdot 10^{8} \mathrm{M}_{\odot}$, and $\Sigma_{g}^{\max } \approx 3.5 \cdot 10^{3} \mathrm{M}_{\odot} \mathrm{pc}^{-2}$. Time evolution shows a moderate decrease of $a_{g}$ and $M_{g}$, whereas $\Sigma_{g}^{\max }$ again remains nearly constant. Shorter lifetimes are found, i.e. $\tau_{g} \approx 0.2 \mathrm{Gyr}$.

\section{Spontaneous Gaseous Bars}

NGC 6946 represents an issue for the above processes: it has a gaseous bar, but no (or very weak) stellar bar! Hence, one is led to wonder if spontaneous gaseous bar instability might occur in some galaxies. Significant self-gravitation $\left(M_{g} \gtrsim 0.3 M_{\mathrm{dyn}}\right)$ would then be required. Such a critical mass could be reached in primordial galactic discs, mergers, and of course stellar bars. However, spontaneous gaseous bars are very unstable (fragmentation). Also, gas self-gravity decreases quickly due to furious star formation. A very short $\tau_{g}$ results. Another appealing possibility for NGC 6946 is that the stellar bar is now nearly dissolved (Friedli \& Benz 1993).

\section{Summary}

The three mechanisms of gaseous bar formation and evolution investigated above show rather short lifetimes $\left(\tau_{g} \approx 1.0 \mathrm{Gyr}\right.$ at most). Typical sizes are $a_{g}=(0.80-0.05) a_{s}$, or $a_{g}=(0.55-0.30) a_{\text {sec }}$. The evolution of $M_{g}$ and $\Sigma_{g}^{\max }$ are essentially controlled by star formation, not by dynamics. This might explain why generally $M_{g} \lesssim 0.3 M_{\mathrm{dyn}}$.

\section{References}

Friedli D., Benz W., 1993, A\&A 268, 65

Friedli D., Martinet L., 1993, A\&A 277, 27

Friedli D., Martinet L., 1997, RevMexAA (Serie de Conferencias) 6, 177

Jogee S., Kenney J.D.P., Smith B.J., 1997, ApJ, in press

Kenney J.D.P., 1996, in: Barred Galaxies, Buta R. et al. (eds.), ASP Vol. 91, p.150

Laine S., 1996, Ph.D. thesis, University of Florida, USA

Martin P, Friedli D., 1997, A\&A 326, 449

Wozniak H., Friedli D., Martinet L., Martin P., Bratschi P., 1995, A\&AS 111, 115 